\title{
Inoculação experimental de Equus asinus com Leishmania chagasi Cunha \& Chagas, 1937
}

\author{
Experimental infection of E quus asinus with \\ L eishmania chagasi Cunha \& Chagas, 1937
}

\author{
Elúzio José Lima Cerqueira',2, Italo Sherlock ${ }^{1}$, Alberto Gusmão², \\ Aryon de Almeida Barbosa Junior ${ }^{1}$ e Maria Nakatani ${ }^{3}$
}

\begin{abstract}
Resumo Quatro Equus asinus foram inoculados com promastigotas de Leishmania chagasi Cunha \& Chagas, 1937 e acompanhados durante 12 meses através de: pesquisa de amastigotas em esfregaços e culturas de sangue periférico em fragmentos de tecido do lábio inferior, medula óssea, baço e fígado e de testes de ELISA e TRALd. Estes foram positivos nos $8^{\circ}, 10^{\circ}$ e $12^{\circ}$ meses após a inoculação. $O$ exame histopatológico pós necropsia, demonstrou discreto número de amastigotas no fígado de dois dos eqüídeos inoculados. Apesar de desafiados com elevado número de promastigotas, os animais não desenvolveram infecções patentes e não infectaram experimentalmente a vetora Lutzomya longipalpis. Os resultados induzem a acreditar que os eqüídeos são desprovidos de importância como reservatórios na cadeia de transmissão da leishmaniose visceral, embora sirvam como boa fonte de alimentação sangüínea e proliferação da vetora Lutzomyia longipalpis.
\end{abstract}

Palavras-chaves: Leishmania chagasi. Infecção experimental. Eqüídeos. Equus asinus. Lutzomyia longipalpis.

\begin{abstract}
Four Equus asinus were challenged with promastigotes of Leishmania chagasi Cunha \& Chagas, 1937, and followed up for 12 months. They were observed by means of direct testing for promastigotes in smears and culture of peripheral blood, fragments from inferior lip, bone marrow, spleen and liver and the immunological assays ELISA and TRALd. The post-necropsy histological examination demonstrated a small number of amastigotes in the liver of two animals. ELISA and TRALd tests were positive at the $8^{\text {th }}, 10^{\text {th }}$ and $12^{\text {th }}$ month after inoculation. The results suggest that the donkeys were able to overcome the experimental leishmanial infection and did not infect the vector Lutzomyia longipalpis in the laboratory. Consequently they can not be considered an important reservoir in the epidemiological chain of transmission of visceral leishmaniasis, although they represent an important blood source for the vector and its proliferation.
\end{abstract}

Key-words: Leishmania chagasi. Experimental infection. Equidae. Equus asinus.

Observações sobre eqüídeos com leishmaniose têm sido feitas desde $1927^{36}$ mas, só recentemente, esses animais foram melhor investigados como hospedeiros de Leishmanias. Na Venezuela ${ }^{3} 561222$, foi encontrada Leishmania braziliensis ${ }^{2}$ em lesões cutâneas de Equus caballus e Equus asinus. No Brasil 10162627 , foram registradas lesões leishmanióticas, também causadas pela Leishmania braziliensis, em Equus asinus e Equus caballus. A investigação através da intradermorreação, realizada em 54 animais, revelou $44,4 \%$ de positividade desse teste cutâneo ${ }^{25}$. $\mathrm{Na}$ análise de 250 amostras de soros de eqüinos, $11,6 \%$ apresentaram reatividade para o ELISA. Os autores sugerem que os eqüinos poderiam estar atuando nessas áreas como reservatórios de leishmaniose cutânea, contribuindo para a manutenção da doença no peridomicílio. Na Bahia, um inquérito, através ELISA em eqüídeos foi positivo para 17 (22\%) dos animais examinados, embora nenhum apresentasse lesão sugestiva de leishmaniose ${ }^{17}$.

Esses achados relativos a Leishmania braziliensis, acrescidos do fato de que eqüídeos estão sempre presentes nas áreas endêmicas de leishmaniose

\footnotetext{
1. Laboratórios de Parasitologia e Entomologia e Laboratório de Histopatologia do Centro de Pesquisas Gonçalo Moniz da Fundação Oswaldo Cruz, Salvador, BA. 2. Faculdade de Farmácia e Escola de Medicina Veterinária da Universidade Federal da Bahia, Salvador, BA. 3. Laboratório de Diagnóstico de Doenças Infecciosas do HUPES/UFBA, Salvador, BA.

Trabalho realizado com financiamento do CNPq Auxílio 000521130-98 e CAPES

Endereço para correspondência: Prof. Elúzio J. L. Cerqueira. CPqGM/FIOCRUZ. Rua Valdemar Falcão 121, Brotas, Salvador, BA

Tel: 5571 356-8785; Fax: 5571 356-4292.

Recebido para publicação em 29/4/2002

Aceito em 29/10/2003
} 
visceral na Bahia, estimulou-nos a investigar sobre a possibilidade desses animais serem também hospedeiros e reservatórios de Leishmania chagasi.
Os resultados dessas observações serão apresentados em uma série de trabalhos que pretendemos publicar, sendo este o primeiro a respeito.

\section{MATERIAL E MÉTODOS}

Exame clínico, inoculação e cepa de Leishmania. Quatro fêmeas sadias de Equus asinus trazidas do Município de Jacobina para Salvador, com 3 e 4 meses de idade, pesando cerca $40 \mathrm{~kg}$ cada, foram mantidas em baia telada, na Escola de Medicina Veterinária da Universidade Federal da Bahia, isoladas de outros animais, sob os cuidados de um tratador. Previamente, esses animais foram submetidos a um exame clínico geral e deles colhido sangue para verificação da negatividade sorológica para Leishmania. Os animais foram em seguida desafiados, por via intravenosa, com um inóculo de promastigotas de Leishmania chagasi (cepa IOCLc2455) em fase estacionária, multiplicadas em meio de cultura LIT. Foram inoculadas $10^{8}$ promastigotas em $1 \mathrm{ml}$ de cultura, por $\mathrm{kg}$ de peso do eqüídeo.

Como controle da infectividade da Leishmania, a mesma cepa foi inoculada em hamsters, por via interperitonial, porém em dosagem menor. Estes animais controles foram necropsiados após 6 meses, tendo-se confirmado a patogenicidade do parasito para o hamster.

Depois do exame clínico inicial, os animais foram submetidos à coleta de $15 \mathrm{ml}$ de sangue para ELISA e TRALd; pesquisa de amastigotas em esfregaços corados, para hemoculturas e reações imunossorológicas.

$\mathrm{Em} 5 \mathrm{ml}$ de sangue colhido da jugular, adicionou-se anticoagulante; após centrifugação, fez-se a separação da camada branca dos leucócitos, que foi semeada em meio de cultura NNN+LIT. Os animais observados foram ainda submetidos à biópsia do lábio superior; sendo parte deste material semeado em meio de cultura e dele também feitos alguns esfregaços em lâminas. De cada fragmento da biópsia, parte era colocada em soro fisiológico com antibiótico e, posteriormente, semeada em meio de cultura; outra parte era preservada em formol a $10 \%$ para estudo histopatológico.

Após a inoculação, esses animais ficaram em observação diária e foram submetidos, de dois em dois meses, à biópsias e a novas coletas de sangue para a realização, como controle, dos mesmos exames acima mencionados, tendo os procedimentos a seguir descritos.

Anestesia e biópsias. Previamente às intervenções invasivas, os animais foram sedados com $2 \mathrm{ml}$ de dormosedan (detomidine hydrochloride), analgésico e sedativo para uso exclusivo em eqüídeos. Fez-se a tricotomia e a assepsia com álcool iodado no local, onde foi feita a biópsia do fígado, baço e medula óssea, por meio de seringa de $10 \mathrm{ml}$ e agulha de $30 \times 8 \mathrm{~mm}$; de cada órgão eram feitos 5 esfregaços.

Para a biópsia do lábio, utilizou-se punch de $5 \mathrm{~mm}$ de diâmetro; do fragmento biopsiado, com parte fez-se cultura e 5 esfregaços em lâminas; outra parte foi colocada em salina com antibiótico (1 ampola de garamicina para $500 \mathrm{ml}$ da solução), mantendo-a entre 4 a $8^{0} \mathrm{C}$ por 12 horas. $\mathrm{O}$ fragmento foi depois transferido para outro tubo contendo meio de cultura e ainda uma terceira parte era colocada em formol a $10 \%$, para estudo histopatológico.

Ainda, foram realizados, com parte dos fragmentos, esfregaços em lâminas, semeio em meio de cultura e inoculação em hamsters.

Pesquisa de amastigotas em esfregaços em lâminas. Para cada animal inoculado, foram confeccionadas cinco lâminas com esfregaço de sangue e dez lâminas com material de biópsia do lábio inferior. Os esfregaços foram corados pelo Panótico Rápido LB ${ }^{\circledR}$ $(\text { LABORCLIN })^{7}{ }^{13}$ para a pesquisa de amastigotas em microscopia óptica. Após a necropsia, procedeu-se da mesma forma para a confecção dos esfregaços dos diversos órgãos.

Cultura para isolamento do parasito. O sangue coletado em tubos com EDTA foi centrifugado, durante 30 minutos a 3.000rpm, separado o soro, acrescentados $5 \mathrm{ml}$ de solução fisiológica e novamente centrifugado, durante 15 minutos a 3000rpm, sendo a camada branca de leucócitos transferida para o meio de cultura.

As biópsias do baço, fígado e medula óssea foram conservadas na própria seringa e colocadas em uma caixa de isopor contendo gelo e, transportadas da Escola de Medicina Veterinária ao Laboratório de Parasitologia e Entomologia (LAPEN) do Centro de Pesquisas Gonçalo Moniz (CPqGM), onde foram semeadas.

As culturas foram observadas a fresco, semanalmente, durante 5 semanas, através de uma gota do material entre lâmina e lamínula, em microscópio óptico. O meio de cultura empregado foi à associação Liver Infusion Triptase (LIT) e Novy-McNeal- Nicolle (NNN).

Exame histopatológico. Fragmentos obtidos após a necropsia do: fígado, baço, medula óssea, pulmão, coração, intestino, músculo liso, músculo estriado e pele da orelha foram fixados em formol a $10 \%$ e submetidas a processamento histológico rotineiro. Cortes de 5 micrômetros, de cada órgão, foram corados pela hematoxilina e eosina e examinados por microscopia de luz.

Teste ELISA. Os soros obtidos por centrifugação, foram mantidos a $-20^{\circ} \mathrm{C}$ até o momento da realização do teste ELISA. A pesquisa de IgG pelo ELISA foi realizada segundo as técnicas descritas ${ }^{8}$. Foram realizados dois tipos de ELISA. No primeiro (ELISA I), foi utilizado como antígeno um lisado crude de Leishmania (Leishmania chagasi lysate) e, no segundo 
(ELISA II), a proteína rk39, ambos expostos a um conjugado anti-horse. Os dois antígenos foram fornecidos pela CORIXA CORP de Seattle, WA, USA. A diluição do soro foi de 1:100, em solução tampão PBS $0.01 \mathrm{M}$ e pH 7,2. O conjugado usado foi o antihorse IgG da SIGMA, A-6917, diluído em 1:10.000, em solução tampão PBS $0,01 \mathrm{M}+1 \%$ Tween $20+0,1 \%$ BSA. O substrato usado foi o TMB Microwell Peroxidase Substrate - Kirkegaard \& Perry Laboratories. As placas de ELISA foram sensibilizadas com as seguintes concentrações: crude $=1 \mathrm{ug} /$ well e rk39 $=25 \mathrm{ng} /$ well, diluídos em tampão carbonato $0,01 \mathrm{M} \mathrm{pH} \mathrm{9,6} \mathrm{com}$ incubação de 15 minutos para cada etapa. A solução STOP foi o ácido sulfúrico $1 \mathrm{~N}$. A solução de lavagem foi o tampão PBS 0,01M pH 7,2 + 1\% Tween 20. Leitura das placas em $450 \mathrm{~nm}$. O valor do cut-off foi calculado a partir da densidade ótica (DO) média de um grupo de 30 amostras de soro de cavalo, mais três desvios padrões. As amostras referidas foram fornecidas pelo Instituto Butantã de São Paulo, após a comprovação de sua negatividade para leishmaniose.
Teste TRALd. O Teste Rápido Antígeno para Leishmania donovani (TRALd) é um teste qualitativo imunocromatográfico in vitro, com alta especificidade para detecção de anticorpos para Leishmanias viscerotrópicas no soro e plasma ${ }^{8}$. O TRALd utiliza como antígeno a proteína rk39, clonada de Leishmania chagasi e proteína A mais ouro coloidal como controle (Manufactured por Health Watch Products, Inc of Stanford, CT, US) 11152324 . O kit tem uma janela para leitura do resultado, com uma área de controle superior (C), uma área de controle inferior (T) e um lugar (S) para colocar uma gota da amostra a ser testada. A presença de anticorpos para Leishmania é indicada pelo aparecimento de distintas linhas púrpuras na janela de leitura, ambas ao nível de (T) e (C). $\mathrm{Na}$ ausência de anticorpos de Leishmania, somente a linha controle, confirma que o teste foi realizado corretamente e o reagente funcionou adequadamente. Portanto, duas linhas indicam um resultado positivo, enquanto uma linha na área controle (C) indica um resultado negativo (Figura 1 ).

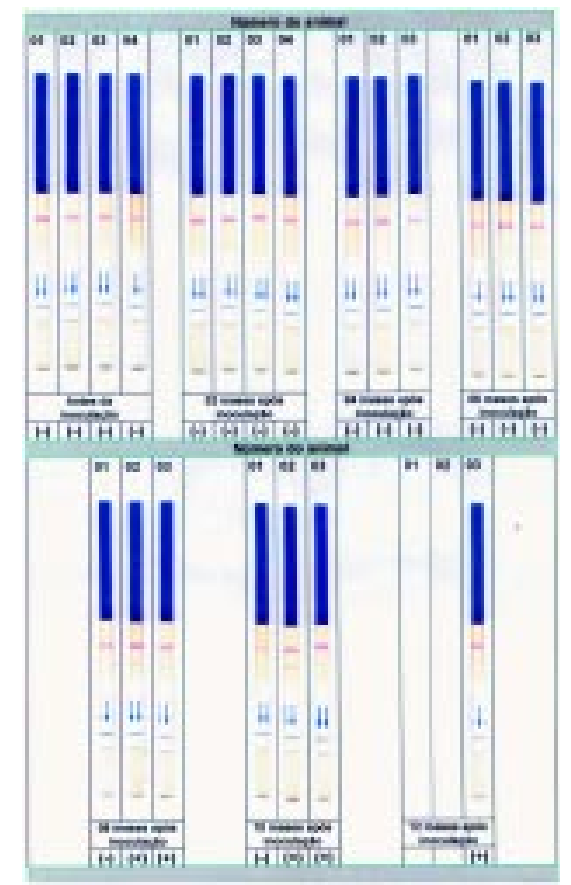

Figura 1- TRALd dos eqüídeos (Equus asinus) inoculados experimentalmente com Leishmania chagasi.

Xenodiagnóstico. Os animais foram submetidos ao xenodiagnóstico com flebótomos, antes e após o desafio com Leishmania, usando-se 30 exemplares fêmeas de L. longipalpis criadas em laboratório. Exemplares de flebótomos dessa colônia foram previamente confirmados como bastante susceptíveis a infecções com L. chagasi. Empregou-se caixinhas especiais contendo aberturas vedadas por malha de nylon fina, seguindo a técnica rotineiramente usada nos Laboratórios de Parasitologia/Entomologia do CPqGM. Os insetos foram dissecados e examinados para flagelados, oito dias após sugarem o animal em observação. Dois meses após a inoculação, foi realizado o primeiro xenodiagnóstico e, daí em diante, foram realizadas nos meses $4,6,8,10$ e 12, final da observação, quando os animais completaram 12 meses de inoculados. 


\section{RESULTADOS}

Após os dois primeiros meses da inoculação, o animal de número 4 adoeceu gravemente sendo por isto sacrificado.

Quanto aos outros três animais, que foram necropsiados 12 meses após a inoculação, apresentavam bom estado nutricional e, embora não mostrassem alterações clínicas aparentes, os seus exames macroscópicos evidenciaram espessamento esbranquiçado disseminado no parênquima hepático, sugestivo de necrose focal; pele, mucosas e tecido subcutâneo pálidos; linfonodos pré-escapulares: aumentados de volume, pálidos e edemaciados. Esôfago: com mucosa pálida. Estômago: regiões glandular e aglandular de tonalidade pálida. Pulmões: superfícies periféricas dos lobos revelando irregularidades, caracterizadas por pequenas áreas elevadas, pálidas e hipercrepitantes (enfisema alveolar multifocal agudo); gordura pericárdica e musculatura cardíaca pálida. Baço: redução de volume total, com discreta consistência ao tato. Superfície externa de tonalidade pálida. Ao corte, discreta reatividade da polpa branca, caracterizada por pontos milimétricos salientes de coloração acinzentada, disseminados com hipertrofia de polpa branca. Fígado: redução de volume total, com superfície lisa. Cápsula e parênquima superficial, revelando lesão cicatricial focal, com aproximadamente 0,5 centímetro de comprimento. Presença marginal, em um dos lobos, de múltiplos pontos milimétricos com tonalidade variando de brancacenta a branco-amarelada, de consistência firme, que se aprofundavam no parênquima superficial (focos calcificados). Bexiga: mucosa pálida. Intestinos delgado e grosso com serosas pálidas. Presença de conteúdo semipastoso a líquido, de tonalidade vermelho-escurecida, no lúmem de todo o trato intestinal.

A pesquisa nos esfregaços foi negativa para amastigotas. As culturas de sangue e de fragmentos obtidos por biópsias também foram negativas para todo o material examinado. Já os hamsters inoculados com a mesma cepa de Leishmania dos eqüídeos e que foram observados durante seis meses, como já nos referimos, apresentaram evolução da infecção leishmaniótica, comprovando a patogenicidade do parasita. Formas amastigotas foram detectadas nos esfregaços corados de seus baços e fígados.

Além do descrito como generalidades, o eqüídeo de número 1 apresentou: fígado com esteatose periportal; baço com acentuada congestão; pele com discreto número de linfócitos e alguns plasmócitos em acúmulos focais. Os demais órgãos (pulmão, coração, músculo liso, músculo estriado esquelético) não apresentaram anormalidades. Não se observou elementos parasitários.

$\mathrm{O}$ animal de número 2 apresentou o fígado com arquitetura geral preservada, hiperplasia das células de Kupffer, acúmulos focais de plasmócitos, necrose hepatocelular focal e granulomas macrofágicos contendo elementos arredondados de 1-5 micrômetros, identificados como amastigotas de Leishmania (Figura 2); baço: com aumento de trabéculas fibrosas e congestão; pulmão: apresentando áreas de espessamento septal; pele da orelha: acúmulos focais de plasmócitos e poucos macrófagos. Os outros órgãos examinados (coração, músculo liso, músculo estriado esquelético) não apresentaram anormalidades.

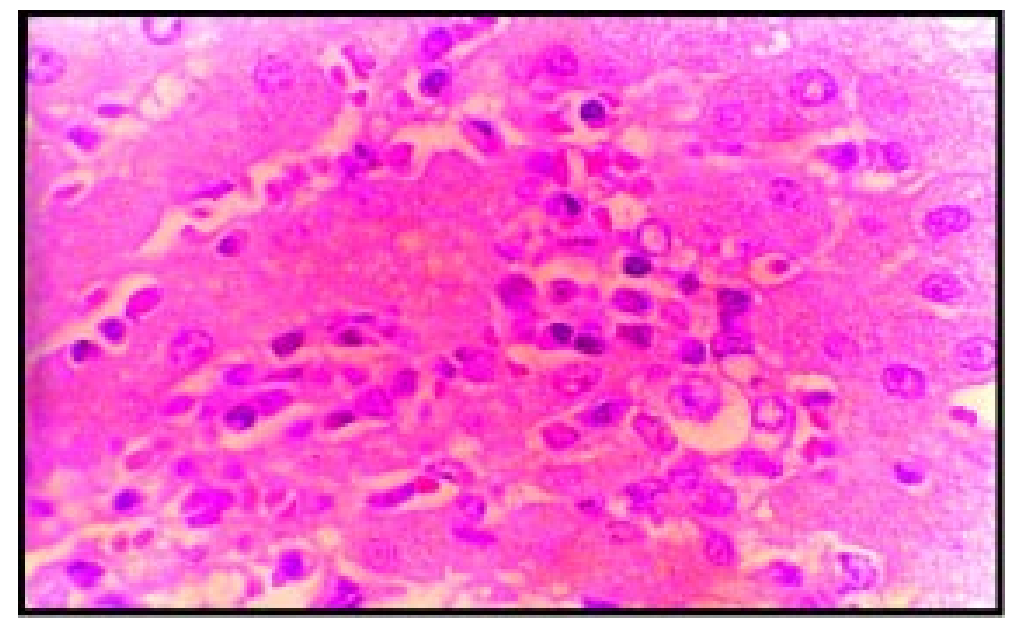

Figura 2 - Grupos de plasmócitos e macrófagos, contendo elementos identificados como Leishmania chagasi. Fígado, animal 2 , HE, 1000X. 
O animal de número 3 apresentou: fígado congesto com arquitetura geral preservada; acúmulos focais de plasmócitos, necrose hepatocelular focal e granulomas macrofágicos, contendo elementos arredondados de 1-5 micrômetros, identificados como amastigotas de Leishmania; baço: congesto; polpa vermelha, polpa branca e septos fibrosos normais; pele do lábio com hiperplasia epitelial, discreto número de mononucleares subjacentes, sugestivo de queilite crônica; pele da orelha: acúmulos focais de plasmócitos e poucos macrófagos.
Os demais fragmentos tissulares dos órgãos (coração, pulmão, tecido adiposo, músculo liso e músculo estriado esquelético) não apresentaram anormalidades.

Os resultados dos testes de ELISA estão relacionados na Tabela 1. Como se constata, o animal numero 1 apresentou resultados negativos em todos os 12 meses da observação.

O TRALd foi positivo para os animais numero 2 e 3 a partir do oitavo mês da inoculação, repetindo-se a positividade nos $10^{\circ}$ e $12^{\circ}$ meses (Figura 1).

Tabela 1 - Resultado do ELISA I e Il para Leishmania chagasi dos eqüídeos inoculados, experimentalmente.

\begin{tabular}{lcccc}
\hline $\begin{array}{l}\text { Número do } \\
\text { animal examinado }\end{array}$ & Cut-off & ELISA I & ELISA II & Positivo/negativo \\
\hline 1 & 0,262 & 0,137 & 0,137 & negativo \\
2 & 0,262 & 0,305 & 0,313 & positivo \\
3 & 0,262 & 0,314 & 0,315 & positivo \\
4 & 0,262 & - & - & - \\
\hline
\end{tabular}

Como já informamos, o animal número 4 foi sacrificado logo no segundo mês da inoculação, porque adoeceu gravemente com outra patologia.
Todos os xenodiagnósticos para Leishmania com o Lutzomya longipalpis foram negativos para os animais inoculados.

\section{DISCUSSÃO}

Nas zonas rurais do Brasil, os eqüídeos são usualmente utilizados como meio de transporte ou de carga, movimentando-se constantemente pelos diversos recantos das localidades endêmicas. São excelentes atrativos e fonte sanguínea para a alimentação dos flebotomíneos, estimulando dessa forma a proliferação desses vetores.

O fato de termos usado animais provenientes da área endêmica de Jacobina, não parece ter interferido na observação pois os referidos animais estavam negativos no controle preliminar e em dois dos três animais observados, foi possível demostrar a infecção através de exames hitopatológicos e testes sorológicos.

O parasitismo das vísceras por Leishmania no fígado de dois dos eqüídeos inoculados demonstrado pelo exame histopatológico, para fins de diagnóstico é relevante pois, a punção desses órgãos pode vir a ter resultado positivo na busca do parasitismo. Também, os testes ELISA e TRALd foram valiosos pois apresentaram $50 \%$ de positividade.

Em relação a escolha do tipo de inóculo, sabe-se que amastigotas são mais infectantes do que as formas promastigotas em hamsters ${ }^{20}$, em cães ${ }^{114} 18 \mathrm{e}$ gatos $^{21}$. No presente trabalho, foram utilizadas as formas promastigotas, por serem elas de mais fácil acesso nas condições que trabalhamos. Não se levou em conta que a alta carga parasitária do inóculo de formas de baixa infectividade poderia contribuir para o aparecimento de uma resposta imune devido a antigenemia promovida pela alta dose do inóculo e baixa infectividade das formas, o que não parece ter ocorrido com o nosso experimento desde que, em dois dos três animais inoculados foi mantida a infecção por um ano, embora com discreto número de parasitas que não conseguiram infectar experimentalmente o vetor.

Relativo à via de inoculação, um trabalho sobre leishmaniose visceral canina experimental ${ }^{18}$ mostrou que a via endovenosa proporcionou taxa de infecção superior à obtida por via intradérmica, independentemente da dose do inóculo. A taxa de infecção obtida nas diferentes doses de inóculo e vias de administração foram variadas nos animais inoculados com culturas de Leishmania chagasi. A dose de $10^{4}$ promastigotas não resultou infectante para os animais que testaram. Os inóculos com $10^{5}$ e $10^{6}$ promastigotas mostraram taxas de infecção semelhantes, sendo que $10^{7}$ promastigotas foi a dose que conseguiu infectar maior $(61,5 \%)$ número de animais. Outros autores conseguiram também infectar cães com doses de inóculo de amastigotas de L. chagasi e L. donovani que variavam de $10^{7}, 10^{8}$ e $10^{10}$ parasitas $/ \mathrm{kg}$ de peso corporal $^{119}$, concluíram que a via endovenosa eram a mais eficaz para infectar os animais com formas amastigotas. Tomamos como base a experiência dos autores citados e optamos pela via endovenosa para a inoculação de $1 \times 10^{8}$ parasitos $/ \mathrm{kg}$ de peso corporal.

Finalmente, com base em nossas observações, concluímos que: 1) A pesquisa de amastigotas em lâminas, cultura e isolamento do parasita e biópsias e 
estudo da medula óssea, do baço e do fígado, apresentaram resultados negativos nos exemplares de Equus asinus observados; 2) Contudo, o parasitismo por Leishmania foi demonstrado, após necropsia aos 12 meses de inoculação, através do exame histopatológico do fígado de dois dos eqüídeos inoculados. Estes dois animais também apresentaram os testes de ELISA e TRALd positivos no $8^{\circ}, 10^{\circ}$ e $12^{\circ}$ meses da inoculação; 3) Parece que os animais inoculados com a elevada carga de promastigotas de Leishmania chagasi, devido a capacidade imunológica do mamífero ou a antigenemia conseqüente a elevada dose do inóculo e baixa infectividade da forma promastigota, foram capazes de amenizar a progressão da infecção, promovendo, espontaneamente, a diminuição da carga parasitária; 4) O escasso parasitismo, na circulação periférica e na pele do animal dificultou a infecção do flebótomo. Isto, provavelmente, torna o animal, sem importância na cadeia epidemiológica como reservatório do parasita; 5) Entretanto, os eqüídeos mantêm certo grau de importância na ecologia da leishmaniose visceral como fonte sanguínea para os flebótomos, possibilitando aumento da reprodução e densidade do vetor, elevando conseqüentemente o risco de transmissão do agente etiológico.

\section{AGRADECIMENTOS}

Os autores agradecem ao Dr. Roberto Badaró, pelas facilidades que promoveu para a realização deste trabalho; ao Dr. Moacyr Paranhos, ao mestrando Evandro Morais Silva, pela colaboração durante a realização das observações; aos técnicos Antônio Carlos dos Santos, do Laboratório de Parasitologia e Entomologia do Centro de Pesquisas Gonçalo Moniz/FIOCRUZ, José Serafim, Zacarias dos Santos e Washington França da Silva, da Escola de Medicina Veterinária da Universidade Federal da Bahia pela ajuda nos exames, manutenção e realização das necrópsias dos animais.

\section{REFERÊNCIAS BIBLIOGRÁFICAS}

1. Abranches P, Silva-Pereira MCD, Conceição-Silva FM, SantosGomes GM, Janz JG. Canine leishmaniasis: pathology and ecological factors influencing transmission of infection. Journal of Parasitology 77: 557-561, 1991.

2. Aguillar $\mathrm{CM}$. Leishmaniasis tegumentária en los caserios Solano y Valle Hondo del Estado Cojedes. Participacion de los animales domésticos. Thesis, Universidad de Carobobo, Valencia, Venezuela, 1985.

3. Aguillar CM, Fernandez R, Fernandez E, Deane LM. Animales domésticos y leishmaniasis tegumentária americana. Acta Cientifica Venezolana 30(supl):121, 1979

4. Aguillar CM, Rangel EF, Deane LM. Cutaneous leishmaniasis is frequent in equines from an endemic area in Rio de Janeiro. Memórias do Instituto Oswaldo Cruz 81:47-472, 1986.

5. Aguillar CM, Rangel EF, Garcia L, Fernandez E, Momen H, Grimaldi GF, Vargas Z. Zoonotic cutaneous leishmaniasis due to Leishmania (Viannia) braziliensis associated with domestic animals in Venezuela and Brazil. Memórias do Instituto Oswaldo Cruz 84:19-28, 1989.

6. Aguillar CM, Rangel EF, Grimaldi GF, Momen H. Human, canine and equine leishmaniasis caused by Leishmania braziliensis braziliensis in an endemic area in the State of Rio de Janeiro. Memórias do Instituto Oswaldo Cruz 82:143, 1987.

7. Andrade SL. Leishmaniose tegumentar americana em área de ocupação recente na periferia da Cidade de Manaus, Estado do Amazonas, Brasil. Tese de Mestrado, Instituto Oswaldo Cruz, Fundação Oswaldo Cruz, Rio de Janeiro, RJ, 1998.

8. Badaró R, Benson D, Eulálio MC, Freire M, Cunha S, Netto EM, Pedral-Sampaio D, Madureira C, Burns JM, Houghton RL, David JR, Reed SG. rK39: a cloned antigen of Leishmania chagasi that predicts active visceral Leishmaniasis. The Journal of Infectious Diseases 173: 758-761, 1996.

9. Barbosa-Santos EGO, Marzochi MCA, Urtado W, Queiroz FJ, Cruz JB, Pacheco R, Momen H. Forma mucocutânea e disseminada da infecção natural por Leishmania (V.) braziliensis em uma égua Equus caballus. I. Apresentação de um caso. In: Resumos dos Anais da IV Jornada Cientifica da Fundação Oswaldo Cruz, Rio de Janeiro, p. 110, 1991.

10. Barretto AC, Vexenat JA, Cassia A, Rosa OC, Cuba CC, Lago E, Peterson NE. Epidemiological study of cutaneous Leishmaniasis in equines from an endemic area of the state of Bahia, Brazil. Memórias do Instituto Oswaldo Cruz 81:63, 1986.

11. Bhatia A, Daifalla NS, Jen S, Badaro R, Reed SG, Sheiky YAW. Cloning, characterization and serological evaluation of k39 and k26: two related hydrophilic antigens of Leishmania chagasi. Molecular Biochimica Parasitology 102: 249-261, 1999.

12. Bonfante-Garrido R, Melendez E, Torres R, Morillo N, Arredondo C, Urdaneta I. Enzootic equine cutaneous leishmaniasis in Venezuela. Transactions Royal Society of Tropical Medicine and Hygiene 75: 471, 1981.

13. Coelho L, Souza JU, Oliveira R. Diagnóstico parasitológico em leishmanioses por método de coloração alternativo. Transactions Royal Society of Tropical Medicine and Hygiene 31(supl I): 172-173, 1998.

14. Dubreil N, Vidor E, Moreau Y. Experimental canine leishmaniasis: A clinical and immunological study. International Congress of Parasitology, Paris, 1990.

15. Evans TG, VasconceloS IAB, Lima JW, Teixeira JM, Mcaullife IT, Lopes UG, Pearson RD, Vasconcelos AW. Canine visceral leishmaniasis in northeast Brazil: Assessment of serodiagnosis methods. American Journal of Tropical Medicine and Hygiene 42:118-123, 1981

16. Falqueto A, Varejão JBM, Sessa PA. Cutaneous leishmaniasis in horse (Equus caballus) from endemic area in the state of Espírito Santo, Brazil. Memórias do Instituto Oswaldo Cruz 82: 443, 1987.

17. Follador I, Araujo C, Cardoso MA, Tavares-Neto J, Barral A, Miranda JC, Bittencourt A, Carvalho EM. Surto de leishmaniose tegumentar americana em Canoa, Santo Amaro, Bahia, Brasil. Revista da Sociedade Brasileira de Medicina Tropical 32: 497-503, 1999. 
18. Genaro O. Leishmaniose visceral canina experimental. Tese de Doutorado, Universidade Federal de Minas, Belo Horizonte, MG, 1993.

19. Keenan CM, Hedricks LD, Lightner L, Webster HK, Johnson A. Visceral leishmaniasis in the German shepherd dog. I. Infection, clinical disease, and clinical pathology. Veterinary Pathology 21: 74-79, 1984.

20. Keitly JS. Infectivity of Leishmania donovani amastigotes and promastigotes for golden hamsters. Journal of Protozoology 23: 244-245, 1976

21. Kirkpatrick CE, Farrell JP, Goldschmid MH. Leishmania chagasi and $L$ donovani: Experimental infections in domestic cats. Experimental Parasitology 58: 125-131, 1984.

22. Mazza S. Leishmaniose cutánea en el caballo y nueva observacion de la misma en el perro. Boletin Instituto Clinica Quirugico 3: 462-464, 1927.

23. Scott JM, Shreffler WG, Ghalib HW, Asad AL, Siddig M, Badaró $R$, Reed SG. A rapid simple diagnostic test for active visceral leishmaniasis. American Journal of Tropical Medicine and Hygiene 44: 272-277, 1991.

24. Singh R, Gilman-Sachs A, Chang KP, Reed SG. Diagnostic and prognostic value of $\mathrm{k} 39$ recombinant antigen in India Leishmaniasis. Journal of Parasitology 81:1000-1003, 1995.

25. Tolezano JE, Taniguchi HH, Barbosa JAR, Araujo MFL, Cunha EA, Bisugo MC, Barbosa JER, Barbosa SCF, Alkimin MGA, Uliana SRB, Foeter-Winter LM, Pedroso FR, Shaw JJ. Epidemiologia da leishmaniose tegumentar americana (LTA). Revista da Sociedade Brasileira de Medicina Tropical 32 (supl II): 126-127, 1999.

26. Vexenat JA, Barretto AC, Rosa ACO, Sales CC, Magalhães A. Infecção natural de Equus asinus por Leishmania brasileense brasiliensis - Bahia, Brasil. Memórias do Instituto Oswaldo Cruz 81: 237-238, 1986.

27. Yoshida ELA, Correa FMA, Marques AS, Stolf HO, Dillon NL, Momen $\mathrm{H}$, Grimaldi Jr G. Human, canine and Equine (Equus caballus) leishmaniasis due to Leishmania braziliensis $(=$ L. braziliensis braziliensis) in the south-west region of São Paulo State, Brazil. Memórias do Instituto Oswaldo Cruz 85: 133-134, 1990. 